\title{
Mesures des déformations bloquées dans un disque de bois vert. Méthode de la fermeture
}

\author{
D Jullien, J Gril \\ Laboratoire de mécanique et génie civil, université de Montpellier-1I, CC 81, \\ place Eugène-Bataillon, 34095 Montpellier cedex 5, France
}

(Reçu le 6 mars 1995 ; accepté le 12 juillet 1995)

\begin{abstract}
Summary-Measurement of locked-in strains in a green crosscut. The closing method. Initiation and propagation of heart checks at log ends after tree felling lead to serious problems for wood exploitation. This phenomenon is caused by the release of strains locked-in during cell maturation and is triggered by steaming; its understanding requires an improved knowledge of residual strains in logs, particularly transversally to fibers. A procedure completing the longitudinal measurements at tree periphery is presented, based on the 'instantaneous' closing of green crosscuts caused by a radial opening, followed by the 'hygrothermal' closing provoked by boiling (fig 3 ). The relative displacement of two pins nailed near periphery is measured using a special device (fig 1) and divided by girth to give a global transverse strain $\varphi$. Local measurements of the radial strain $\varepsilon_{R}$ and the tangential strain $\varepsilon_{T}$ performed using the same device (fig 2) showed that $\varphi$ partially expressed the strain anisotropy $\varepsilon_{\mathrm{T}}$ $-\varepsilon_{R}$ (fig 8). Instantaneous values of $0.2 \%$ obtained on 132 chestnuts (Castanea sativa Mill) were weakly correlated to longitudinal strains (fig 10). Hygrothermal strains reached $0.5 \%$ after $30 \mathrm{~min}$ at $80^{\circ} \mathrm{C}$, correctly expressing splitting trends: the more wood cracked tangentially, the less it did radially, the potiential transverse strain remaining for all rupture modes (fig 12).
\end{abstract}

\section{chestnut / wood mechanics / transverse strain / hygrothermal recovery / heart check}

Résumé - L'apparition et la propagation de fentes radiales en bout de grume suite à l'abattage d'un arbre entraînent de sérieux problèmes pour l'exploitation du bois. On sait que ce phénomène est provoqué par la libération de déformations bloquées lors de la maturation cellulaire et est accéléré parl'étuvage humide. Il est donc nécessaire, pour le comprendre, de mieux connaître les déformations résiduelles dans la grume, notamment transversalement aux fibres. Les auteurs présentent une procédure complétant les mesures longitudinales en périphérie des arbres sur pied, basée sur la fermeture "instantanée " de disques de bois vert suite à la découpe d'une saignée radiale, suivie de la fermeture "hygrothermique " provoquée par l'étuvage en bain-marie. Le déplacement relatif de deux plots cloués près de la périphérie, divisé par la circonférence de la rondelle, mène à la déformation transverse globale. Les valeurs instantanées de 0,2\% obtenues sur 132 châtaigniers (Castanea sativa Mill) sont faiblement corrélées aux déformations longitudinales. Les déformations hygrothermiques atteignent $0,5 \%$ après 30 minutes à $80^{\circ} \mathrm{C}$, traduisant correctement les tendances à la fissuration : plus le bois roule, moins il fend radialement, le potentiel de déformation transverse restant le même quel que soit le mode de rupture.

châtaignier / mécanique du bois / déformation transverse / recouvrance hygrothermique / fissuration à ccur 


\section{INTRODUCTION}

Les opérations de première transformation du bois se heurtent à des problèmes de fissuration et de déformation dont la meilleure maîtrise est un enjeu économique majeur, surtout sur certaines essences très fissiles telles que le châtaignier. L'origine de ces phénomènes, lorsqu'ils se manifestent avant séchage, doit être recherchée dans l'existence de déformations bloquées lors de l'élaboration du matériau et dont les opérations de coupe et d'étuvage stimulent la recouvrance. Les couches de bois formées successivement à la périphérie de l'arbre lors de la croissance tendent à se déformer du fait de la maturation cellulaire, ce qui engendre leur mise sous contrainte et l'apparition de déformations dont une partie reste bloquée dans la matière. Depuis Kübler (1959a, b), on sait analyser le champ de contraintes résultant de ce processus et le risque de fissuration à cœur qui lui est associé (Archer, 1987 ; Fournier et al, 1991). On sait aussi que l'étuvage humide des grumes, souvent indispensable à l'usinage, accélère la recouvrance de déformations bloquées et, de ce fait, amplifie le phénomène de fissuration (Kübler, 1959c, 1987).

L'étude des contraintes de croissance suscite l'intérêt des équipes françaises depuis longtemps (Bedel et Thibaut, 1976 ; Chardin et Bégé, 1982 ; Ferrand, 1982). Depuis quelques années, elle évolue vers une approche globale de la mécanique de l'arbre en relation avec des botanistes, anatomistes et physiologistes (Thibaut, 1992). Jusqu'à présent, l'accent a été mis sur l'étude des déformations longitudinales, qui interviennent plus directement dans les réorientations des tiges et la répartition de bois de réaction, et dont la mesure par la méthode dite du trou unique est maintenant bien au point (Baillères, 1994 ; Fournier et al, 1994). Elle consiste à mesurer le raccourcissement des fibres consécutif au perçage de trous sur toute la péri- phérie. Les déformations tangentielles en périphérie des tiges ont aussi été l'objet d'investigations au Japon (Okuyama et al, 1981) ; il a été ainsi montré que les deux déformations (longitudinale et tangentielle) ne sont pas nécessairement corrélées, et, qui plus est, que les cinétiques de leur mise en place durant une saison de croissance ne coïncident pas. Par ailleurs, des méthodes de caractérisation du champ de déformations bloquées en tout point d'une bille ou d'une rondelle, fondées sur des découpes successives, ont été mises au point mais restent lourdes à mettre en œuvre dans le cadre d'études de variabilité à grande échelle (Sakaki et al, 1981a, b ; Chardin et Bégé, 1982 ; Thomas et al, 1995).

Dans le cadre des campagnes de mesures organisées actuellement pour étudier la relation entre morphologie de l'arbre et contraintes de croissance, il est souhaitable de disposer d'une méthode de caractérisation de l'état des contraintes transverses dans les tiges suffisamment simple et fiable, complémentaire des mesures longitudinales. L'objectif de cet article est la description d'une procédure expérimentale originale permettant d'estimer globalement les déformations transverses bloquées dans une tige. Une série de résultats obtenus sur des peuplements de châtaigniers seront présentés, et confrontés à des mesures longitudinales ou de fissuration.

\section{MATÉRIEL ET MÉTHODE}

\section{Matériel ligneux}

Le bois étudié est issu de taillis de châtaignier (Castanea sativa Mill) récolté dans sept sites français ou italiens. II faut préciser, pour expliquer le protocole expérimental, que les brins choisis ont fait, sur pied et à 1,30 m du sol, l'objet d'une mesure des déformations résiduelles longitudinales selon la méthode du trou unique mentionnée dans l'introduction. Pour chacun des brins, un billon a été prélevé après abattage à proximité de la couronne de mesures, puis une 
rondelle à faces parallèles en a été extraite au moyen d'un dispositif adapté pour scie à ruban. Les rondelles étaient stockées dans l'eau froide et testées au maximum dans le mois suivant l'abattage. Dans le cas de la Bretagne une série de rondelles appariées a été débitée et conservée en parallèle pour des mesures de fissuration. Le tableau I donne pour chaque site, le nombre d'arbres, la plage des diamètres et l'épaisseur des rondelles, et précise si la série de rondelles considérée a été testée sur une ou deux faces et fait ou non l'objet de mesures locales.

\section{Méthode de mesure}

L'estimation des déformations est basée sur la mesure du déplacement relatif de deux plots cloués sur la rondelle, au moyen d'un comparateur Mitutoyo (référence 543-170B) initialement conçu pour la mesure de déplacement axiaux et transformé comme indiqué sur la figure 1. Une série de touches (type 101122) solidaires du comparateur servent de point fixe, tandis qu'une autre touche est solidaire du bras mobile. La tête des plots est munie d'un évidement conique dans lequel peut se positionner l'une de ces touches. Pour une distance initiale donnée $d_{0}$ entre les deux plots, on choisit une touche fixe située à distance $d_{\text {ref }}$ de la touche mobile à sa position d'origine, de sorte que la mesure du comparateur étant $\delta_{0}$, on a $d_{0}=d_{\text {ret }}+\delta_{0}$. Après déformation de la rondelle, on recommence la mesure en prenant bien soin d'utiliser la même touche fixe. La mesure $\delta$ du comparateur correspond donc à une distance $d=d_{\text {ref }}+\delta$, et le déplacement relatif des plots vaut:

$$
d-d_{0}=\left(d_{r e t}+\delta\right)-\left(d_{r e f}+\delta_{0}\right)=\delta-\delta_{0}
$$

La course du bras mobile $(12 \mathrm{~mm})$ étant supérieure à l'intervalle entre les touches fixes $(8 \mathrm{~mm})$, il est en principe possible, avec ce système, de mesurer des petits déplacements par rapport à une gamme continue de distances initiales (de 1 à $15 \mathrm{~cm}$ ). Malgré des effets parasites dus à la flexion de la tige, il a été possible d'obtenir une reproductibilité de $\pm 20 \mu \mathrm{m}$ sur la grandeur $\delta$ lue sur le comparateur, donnant donc $\pm 40 \mu \mathrm{m}$ sur chaque déplacement $d-d_{0}$. Pour la mesure de $d_{\text {ref }}$ intervenant dans l'estimation de la longueur initiale $d_{0}$, une lecture au réglet à $\pm 0,5 \mathrm{~mm}$ s'est avérée suffisante.

\section{Procédure expérimentale}

L'emplacement des deux plots est défini systématiquement de la manière suivante : on trace le plus grand des axes passant par la moelle et correspondant en général à une division en deux parties symétriques de l'échantillon. Le secteur de bois est prélevé de part et d'autre du demiaxe défini par le plus grand rayon. Les plots sont placés aux points $A_{1}$ et $A_{5}$ de la figure 2 . Une fois les plots positionnés, on mesure une première fois leur écartement, puis à la suite de deux actions successives exercées sur la rondelle $:$ i) le perçage d'un trou de $14,5 \mathrm{~mm}$ de diamètre centré à la moelle à l'aide d'une perceuse à colonne et le sciage d'un secteur de matériau de la périphérie au cœur au moyen d'une scie à ruban ; ii) l'étuvage humide à $80^{\circ} \mathrm{C}$ pendant une demiheure dans un bain-marie suivi de son refroidissement (fig 3).

Les variations de cet écartement, observées suite à la coupe et suite à l'étuvage, sont appelées respectivement $f^{c}$ et $f^{\theta}$

Lors de l'opération de coupe, la première entaille libérant les contraintes, le bois se referme sur la lame de scie et la bloque. Pour éviter cela, la coupe doit être effectuée progressivement en réalisant plusieurs traits de scie parallèles afin d'élargir la fente. Signalons qu'un traitement particulier a été appliqué aux échantillons provenant de la Bretagne : à chacun des 24 échantillons ayant subi la découpe et la mesure initiale est associé un échantillon non découpé. Les deux rondelles jumelles subissent alors le même protocole d'étuvage : une demi-heure tous les $10^{\circ} \mathrm{C}$ dans un bain-marie passant de 30 à $100^{\circ} \mathrm{C}$. Pour chaque nouvelle température, on mesure la fermeture sur la rondelle découpée et la longueur des fentes à cœur apparaissant sur la rondelle non découpée.

On définit la déformation transverse globale $\varphi$ comme étant le rapport de la fermeture des lèvres $f$ à l'arc effectif $L$ égal à la circonférence de l'échantillon diminuée de l'arc du secteur prélevé, considérant que le rapprochement des lèvres correspondant à l'élongation de cet $\operatorname{arc} L$. $\varphi$ peut être interprété comme la différence entre les composantes radiale et tangentielle de la déformation libérée dans la rondelle. Cette interprétation est rigoureuse lorsque celles-ci sont homogènes et que l'angle du secteur prélevé est petit (Brémond et Guitard, 1982). On peut penser que $\varphi$ reste dans tous les cas traités une bonne estimation de l'anisotropie moyenne de la 
Tableau I. Descriptif des échantillons étudiés.

\begin{tabular}{lcccccc}
\hline \multirow{4}{*}{ Italie } & $\begin{array}{c}\text { Désignation } \\
\text { du site }\end{array}$ & $\begin{array}{c}\text { Nombre } \\
\text { d'arbres }\end{array}$ & $\begin{array}{c}\text { Diamètre } \\
(\mathrm{cm})\end{array}$ & $\begin{array}{c}\text { Épaisseur } \\
(\mathrm{mm})\end{array}$ & $\begin{array}{c}\text { Mesures } \\
\text { sur deux faces }\end{array}$ & $\begin{array}{c}\text { Mesures } \\
\text { locales }\end{array}$ \\
& Torre & 12 & $14-23$ & 16 & oui & oui \\
& Viterbo & 10 & $12-17$ & 16 & oui & oui \\
& Amiata & 16 & $13-22$ & 16 & oui & oui \\
& Isère & 19 & $13-19$ & 8 & non & oui \\
& Poitou & 30 & $10-21$ & 16 & oui & oui \\
& île-de-France & 20 & $12-21$ & 16 & non & non \\
& Bretagne $^{*}$ & 24 & $12-22$ & 16 & non & non \\
\hline
\end{tabular}

* Protocole particulier : mesure de fissuration en parallèle et paliers de température.

course : $12 \mathrm{~mm}$

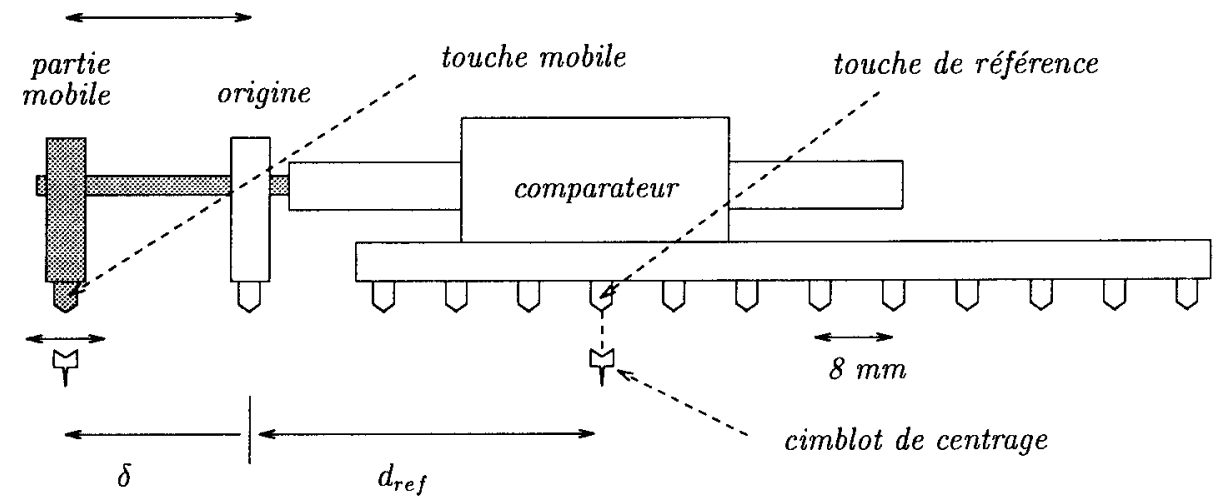

Fig 1. Schéma de principe pour la mesure des déplacements.

déformation induite (Jullien, 1993 ; Jullien et Gril, 1996). La déformation libérée par la coupe est notée $\varphi^{c}$ et celle libérée par l'étuvage $\varphi^{\theta}$ :

$$
\varphi^{\mathrm{C}}=f^{\mathrm{c}} / L \quad \varphi^{\theta}=f^{\theta} / L
$$

où la grandeur $L$ est estimée soit à partir de mesure des diamètres, soit à partir d'évaluation d'arcs.

\section{Estimation de déformations locales}

Dans certains cas, on a choisi d'évaluer également des déformations radiales et tangentielles en utilisant le même mode de mesure et la même procédure expérimentale. L'emplacement des plots supplémentaires est donné par les points $A_{i}$ et $B_{i}$ indiqués sur la figure 2 . Chaque série de mesures $A_{i} B_{i}$ pour $i=1, \ldots 5$ sur la face d'une rondelle nous permet d'accéder à cinq estimations de la déformation radiale moyenne:

$$
\left(\varepsilon_{R}\right)_{i}=\frac{\Delta R_{i}}{R_{i}} \text { avec } i=1, \ldots 5
$$

On peut définir de même quatre déformations tangentielles " apparentes " à partir des distances $A_{i} A_{i+1}$ pour $i=1, \ldots 4$ :

$$
\left(\varepsilon_{T a}\right)_{i}=\frac{\Delta T_{i}}{T_{i}} \text { avec } i=1, \ldots 4
$$

qui sont des combinaisons de la composante radiale $\varepsilon_{R}$ et tangentielle $\varepsilon_{T}$. En faisant l'hypothèse que ces dernières sont homogènes et que les cernes sont circulaires dans la zone de mesure, on obtient par intégration (Gril et al, 1993) :

$$
\varepsilon_{T a}=(1-x) \varepsilon_{T}+x \varepsilon_{R}
$$

avec, dans le cas où le secteur est approximativement un quart de cercle :

$$
x=1-\frac{\pi}{4}
$$


Tableau II. Déformations radiales, tangentielles, transverses moyennes et précisions relatives moyennes correspondantes $(\% \circ)$.

\begin{tabular}{|c|c|c|c|c|c|c|}
\hline & \multicolumn{2}{|c|}{ Radial } & \multicolumn{2}{|c|}{ Tangentiel } & \multicolumn{2}{|c|}{ Transverse } \\
\hline & Élastique & Étuvage & Élastique & Étuvage & Élastique & Étuvage \\
\hline Nombre de données & \multicolumn{2}{|c|}{786} & \multicolumn{2}{|c|}{629} & \multicolumn{2}{|c|}{158} \\
\hline Moyenne & $-0,59$ & $-0,90$ & 1,10 & 3,91 & 2,24 & 5,61 \\
\hline Écart type & 1,17 & 0,70 & 0,58 & 0,87 & 0,45 & 1,06 \\
\hline Précision moyenne & 0,64 & 0,64 & 0,67 & 0,68 & 0,11 & 0,14 \\
\hline
\end{tabular}
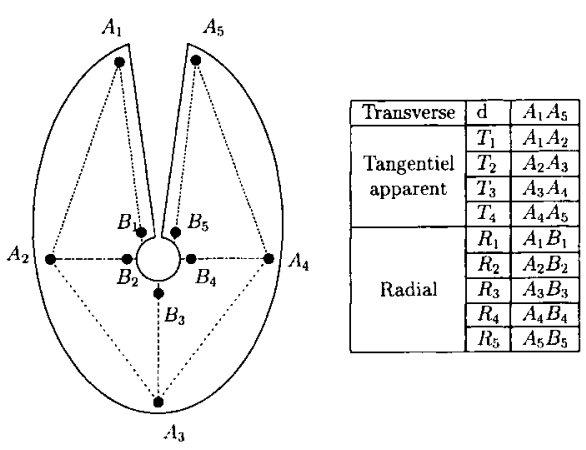

Fig 2. Position des plots et appellations des différentes mesures intervenant dans l'estimation des déformations locales.

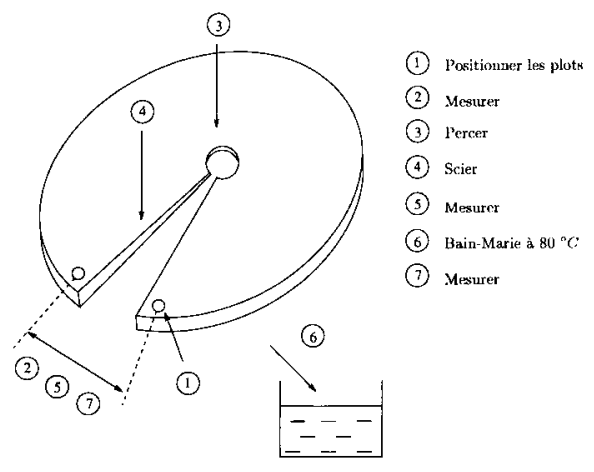

Fig 3. Procédure expérimentale.
On déduit des équations [1] et [3] quatre valeurs de déformation tangentielle corrigée:

$$
\left(\varepsilon_{T}\right)_{i} \approx \frac{1}{1-x}\left(\varepsilon_{T a}\right)_{i}-\frac{x}{1-x} \frac{\left(\varepsilon_{R}\right)_{i}+\left(\varepsilon_{R}\right)_{i+1}}{2}
$$

avec $i=1, \ldots 4$

où l'on a pris pour chaque secteur la moyenne des deux valeurs de $\varepsilon_{R}$ calculées précédemment comme estimateur de la déformation radiale dans la zone de mesure.

\section{Précision des mesures}

Les erreurs moyennes faites sur le calcul des déformations, compte tenu de l'incertitude de mesure sur les mesures de déplacements et de longueurs initiales, sont estimées et présentées dans le tableau II, pour chaque type de déformation. On peut remarquer que, pour les directions radiale et tangentielle, l'erreur moyenne est relativement élevée, tout en restant du même ordre de grandeur que les écarts types respectifs, calculés sur l'ensemble des mesures. Pour la déformation transverse globale, l'erreur ne représente plus que $5 \%$ de la déformation élastique et $3 \%$ de celle d'étuvage, ce qui est très acceptable. II faut ajouter pour cette dernière qu'un essai de répétabilité effectué par ailleurs sur des lots homogènes (deux séries de sept rondelles successives prélevées dans un même tronçon) ont donné, pour la mesure élastique comme hygrothermique, des coefficients de variation inférieurs à $6 \%$.

II s'ensuit que, si la méthode de mesure est assez précise pour la mesure transverse, elle n'est pas vraiment adaptée à l'estimation des déformations radiale et tangentielle du fait de la précision insuffisante par rapport à la base de 
mesure. Nous avons cependant vérifié que les mesures de déplacement effectuées sur chaque face de rondelle restent malgré tout cohérentes entre elles: la figure 4 montre la relation, sur l'ensemble des campagnes, entre la fermeture mesurée et une valeur calculée par reconstitution géométrique à partir des mesures locales.

\section{RÉSULTATS ET DISCUSSION}

\section{Déformation transverse globale}

La figure 5 montre la distribution des déformations transverses globales obtenues pour l'ensemble des mesures, et le tableau III détaille les moyennes et écarts types pour chaque campagne. On a distingué à chaque fois la valeur instantanée due à la coupe de la valeur hygrothermique due à l'étuvage en bain-marie. L'échantillon I5-2 d'Isère n'a pas été pris en compte car il présentait des déformations élevées, sans doute suite à une erreur de mesure. Dans le cas où les deux faces ont fait l'objet de mesures, on a effectué la moyenne des deux. Afin d'intégrer les échantillons de la Bretagne, étuvés par palier de $10^{\circ} \mathrm{C}$, de 30 à $100^{\circ} \mathrm{C}$, on a retenu comme valeur hygrothermique la déformation obtenue à la fin du palier à $80^{\circ} \mathrm{C}$. Malgré les étuvages préliminaires, ces échantillons présentent une

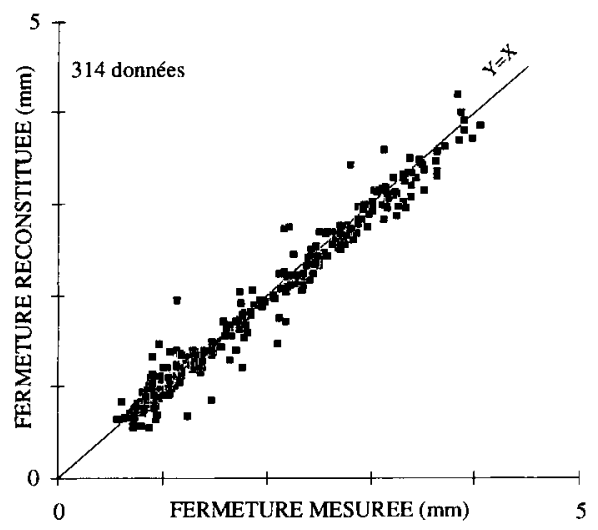

Fig 4. Fermeture reconstituée en fonction de la fermeture mesurée. fermeture du même ordre de grandeur, et même légèrement plus faible, que les échantillons des autres campagnes. En revanche les échantillons issus du site île-deFrance se singularisent par des déformations nettement plus faibles, tant par les valeurs instantanées que par les valeurs hygrothermiques. Les moyennes des autres sites sont proches les unes des autres ; toutefois une analyse de la variance n'a pas permis d'admettre leur égalité. Dans tous les cas, une fermeture a été observée : la déformation instantanée moyenne de $0,2 \%$ est du même ordre de grandeur que la déformation tangentielle périphérique mentionnée dans la littérature
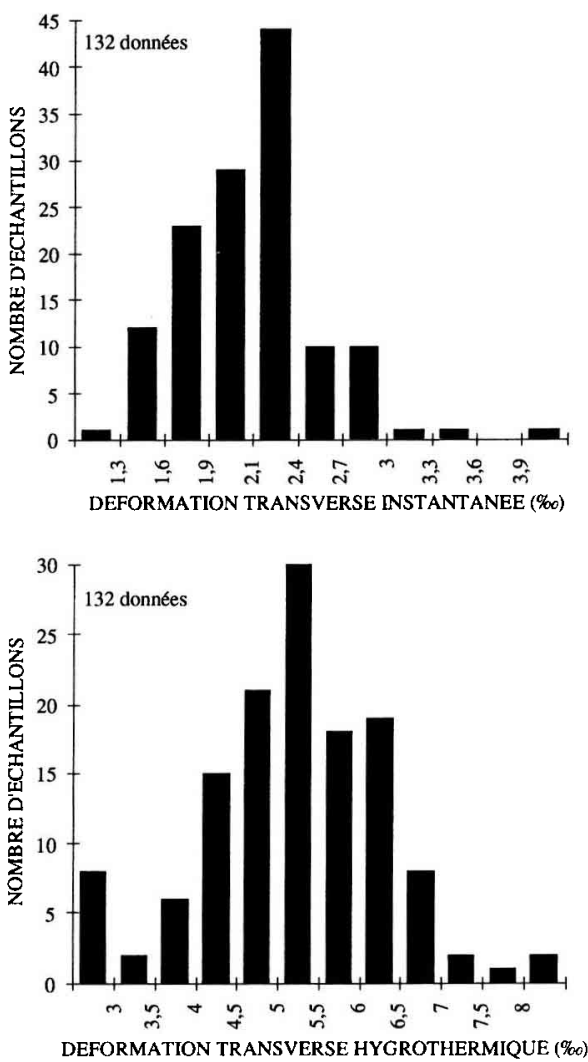

Fig 5. Distribution des déformations transverses instantanée et hygrothermique. 
Tableau III. Caractéristiques des déformations obtenues par campagne $(\% \circ)$.

\begin{tabular}{lccccc}
\hline Campagne & $\begin{array}{c}\text { Moyenne } \\
\text { instantanée }\end{array}$ & $\begin{array}{c}\text { Écart type } \\
\text { instantané }\end{array}$ & $\begin{array}{c}\text { Moyenne } \\
\text { hygrothermique }\end{array}$ & $\begin{array}{c}\text { Écart type } \\
\text { hygrothermique }\end{array}$ & $\begin{array}{c}\text { Nombre } \\
\text { de données }\end{array}$ \\
\hline Torre & 2,32 & 0,59 & 5,38 & 0,92 & 12 \\
Viterbo & 2,30 & 0,25 & 6,24 & 0,90 & 10 \\
Isère & 2,07 & 0,34 & 5,40 & 0,78 & 18 \\
Amiata & 2,03 & 0,22 & 5,21 & 0,78 & 16 \\
Poitou & 2,32 & 0,35 & 5,58 & 0,93 & 30 \\
Île-de-France & 1,75 & 0,31 & 3,82 & 1,55 & 20 \\
Bretagne & 2,07 & 0,29 & 4,81 & 0,70 & 24 \\
Toutes mesures & 2,11 & 0,39 & 5,13 & 1,17 & 132 \\
& & & & &
\end{tabular}

(Archer, 1986) ; la déformation hygrothermique de $0,5 \%$ est légèrement inférieure à ce que les résultats de Gril et al (1993b) sur le châtaignier auraient laissé prévoir.

La relation entre les valeurs hygrothermiques et instantanées (fig 6) est significative au seuil de $1 \%$. La faible valeur du coefficient de corrélation $(r=0,44)$ montre que la déformation instantanée n'explique qu'une partie de la déformation hygrothermique. Cela peut s'interpréter par le fait que la recouvrance hygrothermique correspond surtout aux déformations bloquées durant la maturation cellulaire alors que la recouvrance instantanée exprime, pour une plus grande part, les effets récents de la croissance de l'arbre (Kübler, 1987 ; Gril et Thibaut, 1994). Cette figure

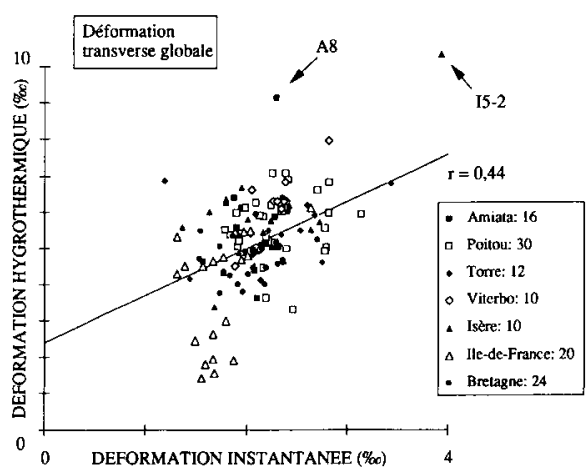

Fig 6. Relation entre déformations tangentielles instantanée et hygrothermique. permet aussi de préciser l'origine des faibles moyennes des échantillons d'île-deFrance : sur les 20, 8 présentent des valeurs hygrothermiques anormalement faibles par rapport aux autres. Aucune explication, notamment au niveau de la morphologie de l'arbre ou des conditions de croissance, n'a pu être avancée pour expliquer cette différence.

\section{Déformations locales par secteur}

Sur certaines campagnes mentionnées dans le tableau I on dispose pour chaque rondelle de quatre évaluations " locales" de la déformation tangentielle $\left(\varepsilon_{T}\right)_{i}$ et cinq évaluations de la déformation radiale $\left(\varepsilon_{R}\right)_{i}$. Une des mesures radiales de l'échantillon PC6-6 provenant de la région Poitou-Charentes s'étant avérée aberrante, seules les mesures transverses de cette rondelle sont conservées. Le tableau II présente les moyennes et écarts types obtenus sur l'ensemble des échantillons et en distinguant à chaque fois les valeurs instantanées des valeurs hygrothermiques. Étant donné la grande dispersion de ces grandeurs locales, une étude statistique s'est avérée indispensable à leur exploitation. Une évaluation locale de la déformation radiale pour le secteur $i$, donnée par la moyenne des déformations radiales $\left(\varepsilon_{R}\right)_{i}$ et $\left(\varepsilon_{R}\right)_{i+1}$ des deux rayons qui le bordent, permet de mettre en relation les déformations radiales et 
tangentielles d'un même secteur. Ces grandeurs, instantanées ou hygrothermiques, ne semblent pas fortement corrélées entre elles. Seule la relation entre les déformations tangentielles instantanée et hygrothermique est significative au seuil de $1 \%$ (fig 7 ), mais le coefficient de corrélation est néanmoins très faible $(r=0,19)$.

\section{Relation entre les déformations locales et la mesure de la fermeture}

La figure 8 met en évidence les relations entre la déformation transverse globale $\varphi$ et la moyenne, pour chaque échantillon, de la différence $\varepsilon_{T}-\varepsilon_{R}$ évaluée pour les quatre quartiers. Il est possible d'imposer à la droite de corrélation de passer par l'origine $(r=0,60)$ dans le cas des valeurs hygrothermiques, mais non dans le cas des valeurs instantanées. La mauvaise corrélation est explicable par la faible précision des mesures pour la déformation radiale, cela étant d'autant plus vrai des valeurs instantanées. D'ailleurs, la relation entre les déformations transverse et tangentielle (fig 9) est plus significative, et toujours plus forte pour les valeurs hygrothermiques $(r=0,82)$ que pour les valeurs instantanées $(r=0,56)$, alors que nous n'avons pas

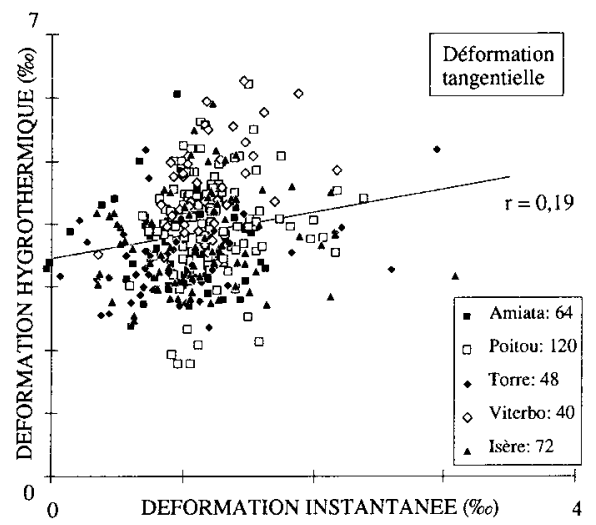

Fig 7. Relation entre déformations tangentielles hygrothermique et instantanée. pu mettre en évidence une relation significative entre les déformations transverse et radiale. Rappelons que, dans le cas d'un champ de déformations induites homogène dans une rondelle axisymétrique, $\varphi$ est proportionnelle à $\varepsilon_{T}-\varepsilon_{A}$ (Brémond et Guitard, 1982). La corrélation relativement bonne observée dans le cas hygrothermique entre $\varphi$ et $\varepsilon_{T}-\varepsilon_{R}$ peut ainsi être reliée à la quasi homogénéité de la recouvrance hygrothermique du bois vert dans la section de la bille (Kübler, 1987), vérifiée précisément sur le châtaignier par Gril et al (1993b). Pour les valeurs instantanées en revanche, les données expérimentales sur
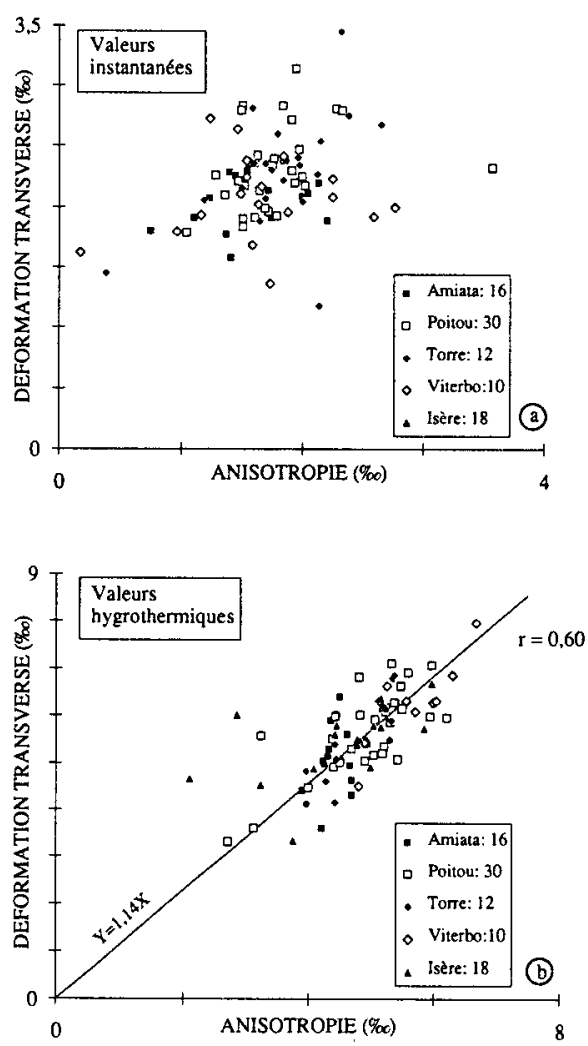

Fig 8. Relation entre la déformation transverse et l'anisotropie $\varepsilon_{T}-\varepsilon_{R}:$ (a) valeurs instantanées et (b) hygrothermiques. 
le châtaignier font défaut, mais si l'on se réfère aux modèles habituels, on s'attend à des répartitions très éloignées de la distribution homogène (Archer, 1987).

\section{Relation avec les déformations longitudinales instantanées mesurées en périphérie de l'arbre sur pied}

Les mesures de déplacement faites sur l'arbre sur pied avec la méthode du trou unique sont converties en déformations longitudinales (Uzielli, 1994). La relation entre les déformations instantanées longitudinale et transverse est significative au seuil de $1 \%$ (fig 10). La faible corrélation
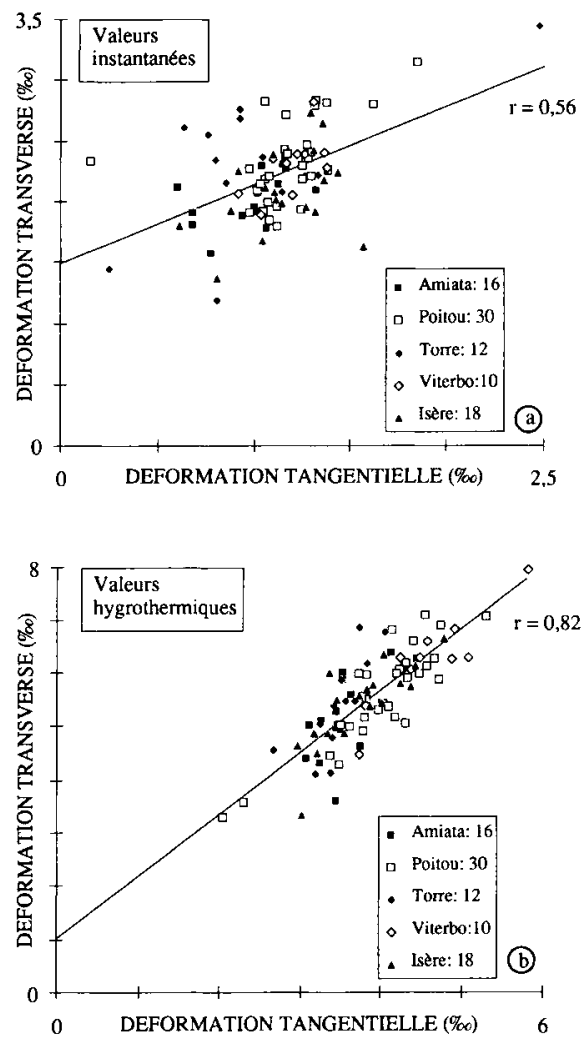

Fig 9. Relation entre les déformations transverse et tangentielle (a) instantanées et (b) hygrothermiques. $(r=0,33)$ peut s'expliquer par le fait que la déformation transverse globale intègre des déformations radiale et tangentielle de tout l'échantillon et témoigne donc de toute l'histoire de l'arbre. À quoi s'ajoute l'absence de nécessité physique pour que les déformations de maturation longitudinale et transverse soient corrélées ; en particulier, il n'est pas prouvé que le bois de tension dans lequel la déformation longitudinale est plus forte soit également associé à une recouvrance tangentielle instantanée élevée (Okuyama et al, 1986).

\section{Relation avec la tendance à la fissuration}

La non-compatibilité entre la géométrie du billon et les déplacements engendrés par la libération de déformations provoque ou amplifie le phénomène de fissuration. Cette non-compatibilité est due à la différence entre déformation tangentielle $\varepsilon_{T}$ et radiale $\varepsilon_{R}$; en d'autres termes, le périmètre et le rayon d'un cercle ne peuvent pas varier indépendamment (Guitard, 1987 ; Berrada, 1991). Dans le cas de la libération des contraintes de séchage $\left(\varepsilon_{T}-\varepsilon_{R}<0\right)$, tout se passe comme si des fentes partant de la périphérie se développent de telle sorte que le rayon reste constant pendant que la quantité de matière sur un périmètre

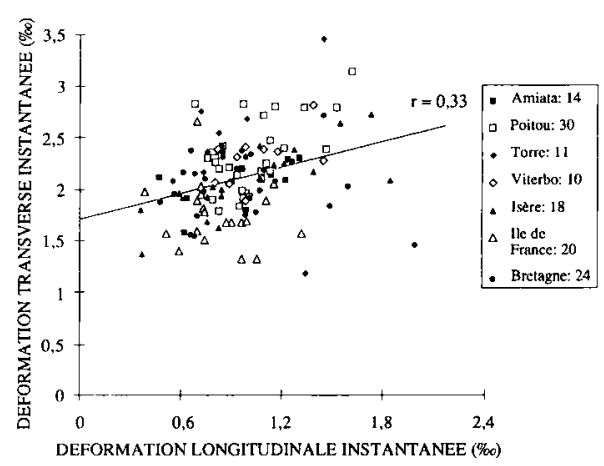

Fig 10. Relation entre les déformations longitudinale et tangentielle instantanée. 
diminue. Dans le cas de la libération des contraintes de croissance $\left(\varepsilon_{T}-\varepsilon_{R}>0\right)$, il existe deux principaux modes de fissuration (fig 11) : i) fissuration radiale ou fentes à cœur en étoile : pour un périmètre extérieur fixé, chaque rayon diminue de longueur ; ii) fissuration tangentielle ou roulure: les cernes se désolidarisent les uns des autres de telle sorte que leur périmètre extérieur reste constant pendant que la matière cumulée sur un rayon diminue. Comme indiqué précédemment la déformation transverse $\varphi$ est une indication globale de l'anisotropie des déformations dans le plan transverse $\varepsilon_{T}-\varepsilon_{R}$. On s'attend donc à une bonne relation entre $\varphi$ et un indice de fissuration des rondelles.

Lors des essais d'étuvage à différentes températures réalisés sur les échantillons provenant de la Bretagne, nous avons remarqué que les rondelles présentant des roulures avant l'expérimentation étaient beaucoup moins sujettes à la formation de fentes. Afin de mieux comprendre le phénomène, nous avons distingué trois catégories de rondelles : très roulées, peu roulées, et non roulées, cette classification étant opérée avant l'étuvage. La figure 12 montre que les deux seuls échantillons qui n'ont pas fendu à l'étuvage présentaient justement des déformations transverses importantes : la grande proportion de roulure préexistante a permis les déplacements dus à la libération des déformations sans rupture supplémentaire du matériau. Pour les sept autres échantillons très roulés la longueur des fentes reste assez petite jusqu'à $80^{\circ} \mathrm{C}$ pour ensuite se propager brusquement : jusqu'à un certain niveau de déformation $(0,7 \%)$, les roulures permettent les déplacements ; au-delà ces derniers sont trop importants et le matériau se fend à cceur. Pour les cinq échantillons peu roulés, les roulures et les fentes existant au départ sont compatibles avec les déformations libérées entre 30 et $50^{\circ} \mathrm{C}$. Au-delà,

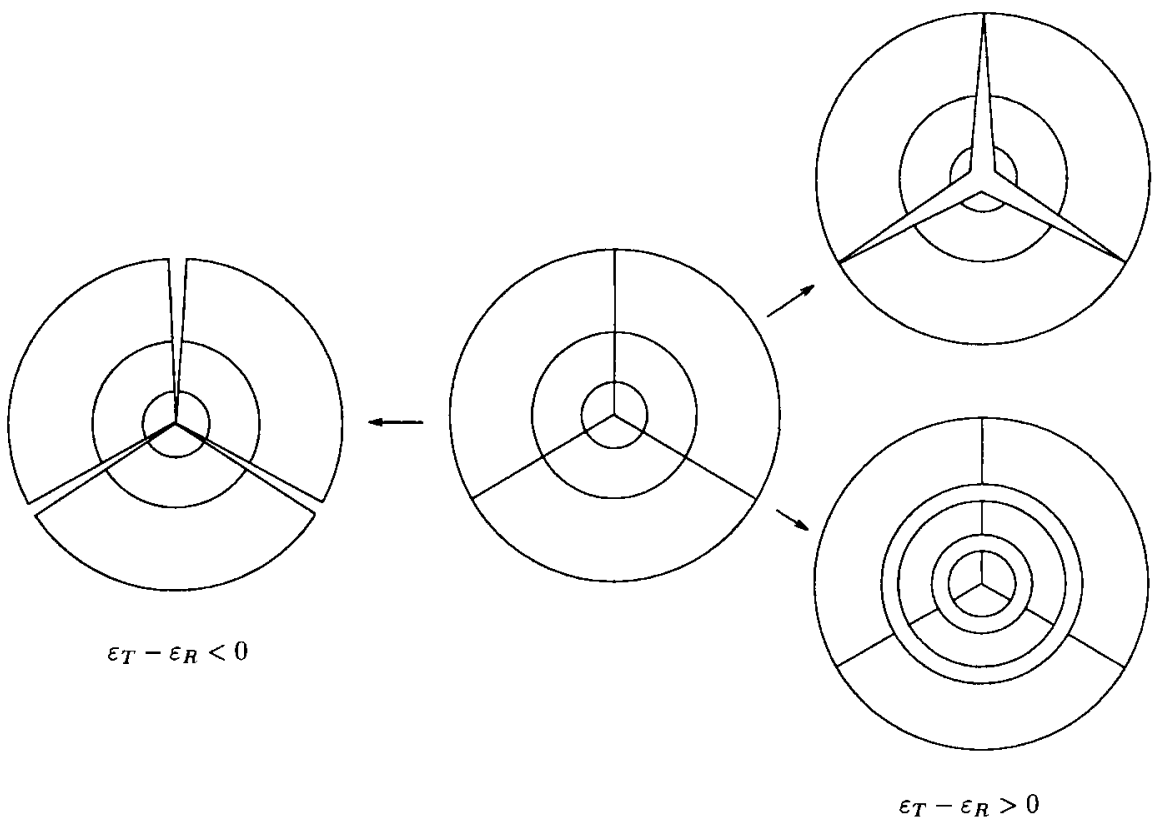

Fig 11. Les principaux modes de fissuration qui aboutissent à la relaxation totale des contraintes de séchage $\left(\varepsilon_{T}-R_{<}<\right)$et des contraintes internes $\left(\varepsilon_{T}-R>0\right)$ 


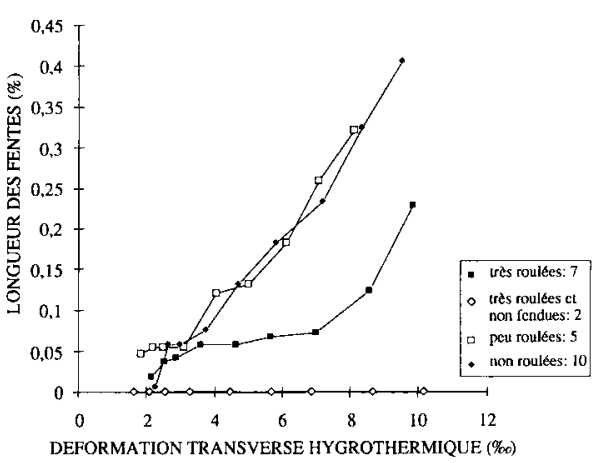

Fig 12. Relation entre la déformation transverse et la longueur des fentes au cours de l'étuvage humide. Chaque point de mesure représente une moyenne par groupe (très roulées, très roulées et non fendues, peu roulées et non roulées). Les chiffres dans la légende représentent les effectifs par groupe. Le premier point correspond à la mesure après la coupe et les suivant aux températures allant de 30 à $100{ }^{\circ} \mathrm{C}$ avec un incrément de $10^{\circ} \mathrm{C}$.

les fentes se propagent proportionnellement à la déformation transverse qui n'excède pas $0,8 \%$. Pour les dix échantillons non roulés la longueur des fentes est quasiment toujours proportionnelle à la déformation transverse qui atteint dans ce cas $0,95 \%$. L'apparition et la propagation des fentes au cours de l'étuvage ne dépendent donc pas seulement de la déformation transverse mais aussi de l'état initial de la rondelle. Lorsque la rondelle présente des roulures, elle a moins tendance à se fendre mais la déformation transverse mesurée n'en est pas pour autant diminuée. La mesure de la fermeture traduit donc le niveau de déformation libérée et non directement le risque de fissuration.

\section{CONCLUSION}

Évaluer la fermeture relative du périmètre d'une rondelle (appelée déformation transverse) suite à une découpe radiale et à un processus d'étuvage semble un bon moyen de comparer des échantillons du point de vue de l'intensité globale des déformations transverses bloquées dans la bille.

Cette méthode présente l'avantage de pouvoir être utilisée aussi bien pour les mesures de déformation instantanée que pour celles de déformation hygrothermique et d'être simple et rapide à mettre en cuvre à grande échelle. Dans tous les cas la précision obtenue pour la mesure est satisfaisante.

L'étude sur le châtaignier montre que la déformation transverse n'est que faiblement liée à la déformation longitudinale instantanée. Elle s'avère être un bon indicateur de l'anisotropie moyenne lorsque les déformations bloquées sont relativement homogènes, comme c'est le cas pour les valeurs hygrothermiques. De plus, l'anisotropie des déformations étant à l'origine de l'apparition et la propagation des fentes à cœur, tout au long du processus d'étuvage, la déformation transverse reste proportionnelle au degré de fissuration de la rondelle. Cependant, ceci n'est plus vrai lorsque les échantillons présentent des roulures au départ : dans ce cas la déformation croît toujours avec la température mais les fentes se propagent beaucoup moins.

Actuellement une étude numérique simulant cette expérimentation est en cours ; elle a pour but d'obtenir un plus grand nombre d'informations sur la sensibilité de la mesure à certains paramètres. Elle devrait permettre de tester l'influence de l'hétérogénéité des déformations sur la fermeture et étudier plus en détail l'influence d'une excentricité de la moelle. On peut également faire varier localement les propriétés rhéologiques du matériau et tester ainsi l'influence de secteurs de bois de réaction par exemple (Jullien et Gril, 1996).

\section{RÉFÉRENCES}

Archer RR (1986) Growth Stresses and Strains in Tree. Springer Verlag, $240 p$ 
Archer RR (1987) On the origin of growth stresses in trees. Part 1. Micro mechanics of the developing cambial cell wall. Wood Sci Technol 21, 139-154

Baillères H (1994) Précontraintes de croissance et propriétés mécano-physiques de clones d'eucalyptus (Pointe Noire, Congo) : hétérogénéités, corrélations et interprétations histologiques. Thèse de doctorat, université Bordeaux-I

Bedel J, Thibaut B (1976) Étude expérimentale du champ des contraintes résiduelles longitudinales dans un billon de bois. Rapport Bois massif reconstitué $n^{\circ} 1$, CTFT

Berrada E (1991) Recouvrance hygrothermique du bois vert. Thèse de doctorat, université Montpellier-II, $281 p$

Brémond C, Guitard D (1982) Apparition des contraintes internes dans les matériaux à symétrie orthotrope cylindrique. Cas des contraintes internes dans le

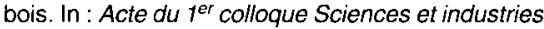
du bois, vol 1, 147-157

Chardin A, Bégé $P$ (1982). Détermination de la composante longitudinale du champ des contraintes de croissance dans des essences métropolitaines et guyanaises. In : Actes du $f^{\text {er }}$ colloque sciences et industries du bois, vol 1, 159-173

Ferrand JC (1982) Étude des contraintes de croissance. 1. Méthode de mesure sur carottes de sondage. Ann Sci For 39, 187-218

Fournier M, Chanson B, Guitard D, Thibaut B (1991) Mécanique de l'arbre sur pied: modélisation d'une structure en croissance soumise à des chargements permanents et évolutifs. 1. Analyse des contraintes de support. Ann Sci For 48, 513-525

Fournier M, Chanson B, Thivaut B, Guitard D (1994) Mesures des déformations résiduelles de croissance à la surface des arbres. Observations sur différentes espèces. Ann Sci For 51, 249-266

Grill J, Barrada E, Thibaut B (1993) Tree mechanics and wood mechanics: relating hygrothermal recovery of green wood to the maturation process. Ann Sci For $51,329-338$

Guitard D (1987) Mécanique du matériau bois et composites. Collection Nabla, Cepadues Édition, $238 \mathrm{p}$

Jullien D (1993) Évaluer les déformations du bois dans le plan transverse : validation d'une méthode. In :
Architecture, structure, mécanique de l'arbre, cinquième séminaire interne, 174-187

Jullien D, Gril J (1996) Numerical analysis of residual stresses remaining in a green wood crosscut after V-cutting and heatting. In : Proc of 3rd Biennal Joint Conference on Engineering System Design and Analysis, Montpellier 1-4 July 1996, PD vol 77, vol 5, 205-212

Kübler H (1959a) Studies on growth stresses in trees. 1. The origin of growth stresses and the stresses in transverse direction. Holz als Roh- und Werkstoff 17 1-9

Kübler H (1959b) Studies on growth stresses in trees. 2. Longitudinal stresses. Holz als Roh-und Werkstoff 17, 44-54

Kübler H (1959c) Studies on growth stresses in trees 3. Effect of heat treatment on the dimensions of green wood. Holz als Roh- und Werkstoff 17, 77-86

Kübler $\mathrm{H}$ (1987) Growth stresses in tree stems and related wood properties. For Prod Abstr 10, 61-119

Okuyama T, Okuyama $T$, Sasaki $Y$, Kikata $Y$, Kawai N (1981) The seasonal change in growth stress in the tree trunk. Mokuzai Gakkaishi 27, 350-355

Okuyama T, Kawai A, Kikata Y, Yamamoto H (1986) The growth stresses in reaction wood. In : Proc 18th $1 U$ FRO World Congress Div 5, 249-260

Sasaki Y, Okuyama T, Kikata Y (1981a) Determination of the residual stress in a cylinder of inhomogeneous anisotropic material. I. Mokuzai Gakkaishi 27, 270276

Sasaki Y, Sakaki Y, Okuyama T, Kikata Y (1981b) Determination of the residual stress in a cylinder of inhomogeneous anisotropic material. II. Mokuzai Gakkaishi 27, 277-282

Thibaut B (1992) Bilan des quatre années du programme. In : Architecture, structure et mécanique de l'arbre, cinquième séminaire interne, 1-20

Thomas R, Fournier M, Thibaut B, (1995) Estimation des contraintes de croissance dans une rondelle de bois. In : Architecture, structure et mécanique de l'arbre, $B^{e}$ séminaire interne, $87-110$

Uzielli $L$ (1994) Nouvelles méthodes de sylviculture et de technologie innovative pour la volorisation du bois de châtaignier comme matière première pour l'industrie. Rapport du projet Forest CEE, Contrat MA2B-CT91-0027 